\title{
Implementing cancer-screening programs by training primary care physicians in India - Findings from the National Institute of Cancer Prevention Research Project ECHO for Cancer Prevention
}

\section{Prajakta Adsul}

University of New Mexico Comprehensive cancer centre

\section{Suzanne Tanya Nethan}

National Institute of Cancer Prevention and Research

\section{Sasha Herbst deCortina}

University of California Berkeley School of Public Health

\section{Kavitha Dhanasekaran}

National Institute of Cancer Prevention and Research

Roopa Hariprasad ( $\square$ roopaicmr@gmail.com )

National Institute of Cancer Prevention and Research https://orcid.org/0000-0003-2032-3432

\section{Research}

Keywords: Capacity building, primary care physicians, cancer screening, project ECHO

Posted Date: August 18th, 2020

DOI: https://doi.org/10.21203/rs.3.rs-57393/v1

License: (a) (i) This work is licensed under a Creative Commons Attribution 4.0 International License. Read Full License

Version of Record: A version of this preprint was published at Global Implementation Research and Applications on January 12th, 2022. See the published version at https://doi.org/10.1007/s43477-021-00032-1. 


\section{Abstract}

\section{Background}

In an effort to address the growing cancer burden, the Government of India introduced a national program for screening and prevention of the most common cancers (oral, breast, and cervical) in 2016. To support the screening program, the National Institute for Cancer Prevention Research (NICPR) adopted the Project ECHO (Extension for Community Healthcare Outcomes) model for training health care providers in cancer screening. Very few studies examine the impact of the ECHO model on provider behavior or health outcomes related to cancer screening. We assess the change in knowledge and, skills among primary care physicians attending NICPR ECHO trainings and the impact of the training program on the implementation of cancer screening services.

Methods

Prior to the start of the online phase and upon completion of the 14 weeks, trainees answered a 23-item questionnaire (administered online via SurveyMonkey). We conducted a descriptive and bivariate analysis of the pre-post assessments conducted on trainees participating in the online phase and where available report on the weekly quizzes and the hands-on workshop assessments.

Results

Six hundred forty-one medical officers have participated in the trainings, across nine cohorts of trainees and this study presents data from 116 primary care physicians that completed both the pre- and post-assessments, trained from May 2019 to February 2020. Almost two-thirds had completed medical training (MD equivalent) $(69.7 \%)$ and $85 \%$ were working in government healthcare facilities. Trainees reported statistically significant improvements before and after the online phase, when queried specifically on knowledge and skills using visual vignettes about oral and cervical cancer screening. Trainees did not report significant changes in the provision of cancer-screening services after completing the program.

\section{Conclusions}

Study findings support the effectiveness of the training program in reaching primary care physicians across the country and improving their knowledge and skills related to screening for breast, oral, and cervical cancer. After the training, very few primary care physicians mentioned implementing cancer screening services highlighting barriers that require further study and development of complementary implementation strategies. Study findings could inform the development and refinement of training for cancer-screening programs in low- and middleincome countries.

\section{Contributions To The Literature}

- Research suggests that providers and health care systems in India do not have sufficient knowledge, training, and resources, to deliver cancer-screening services. To implement the national cancer-screening programs, training primary care physicians is an urgent need in India. 
- We examine the impact of using the ECHO model to train primary care physicians. Our findings show and improvements knowledge and skills, but limited changes in terms of implementation of services in their practice settings

- These findings highlight the training-to-practice gap, recognized in the literature. We outline recommendations for future implementation efforts for national implementation of cancer screening services in India

\section{Background}

Non-communicable diseases are responsible for a large proportion of mortality and morbidity and continue to be an important public health problem.(1) Every year approximately 1,157,294 individuals are diagnosed and 784,821 die due to cancer in India. Breast, oral, and cervical cancer are the three most common cancers occurring in India and account for $34 \%$ of all cancers within the country. (2) For these cancers, screening tests have been demonstrated to help with early detection and thereby prevent mortality due to cancer. The effect on mortality, however, is dependent on widespread access and uptake of cancer screening services (i.e. implementation of cancer screening service) in the population. In an effort to address this burden, the Government of India's Ministry of Health and Family Welfare (MoHFW) introduced a national program for screening and prevention of the most common cancers (oral, breast, and cervical) in 2016. (3)

In India, the central government is mainly responsible for developing national standards and sponsoring key programs, while states hold the primary responsibility for healthcare delivery. The district links the state and the local health center and coordinates with state governments for program implementation. It is estimated that approximately $50 \%$ of the population in India receives care in the private sector, which is fragmented and does not provide contextual uniformity for studying quality of care delivery. $(4,5)$ The other half of the population receives care through the public health care system, depicted in a pyramidal structure (see Figure 1). At the base, primary health care centers and sub-centers have direct interface with the community and are the primary screening facilities. The subsequent tiers represent the referral pathway after patients are screened positive, made up of the community health centers and/or district hospitals for diagnostic evaluations and tertiary level hospitals for specialty care. According to the National Health Profile for India in 2015, each community health center was tied to approximately 5-10 sub centers in the communities across India. (6)

The Indian Council of Medical Research - National Institute of Cancer Prevention and Research (ICMR-NICPR) is the central organization leading and supporting efforts directed at implementation of organized, populationbased, cancer-screening programs across India. NICPR promotes the integration of basic, clinical, and applied research for cancer prevention and control towards the goal of achieving population level outcomes. Previous research in the context of cancer screening suggests that providers and health care systems in India do not have sufficient knowledge, training, and resources to deliver cancer-screening services. (7-10) One of NICPR's primary activities is to support the implementation of the national cancer control plan through training of health care providers (i.e. primary care physicians, gynecologists, nurses, and community health workers) in cancer screening and community-level cancer management.

In 2016, NICPR adopted the Project ECHO (Extension for Community Healthcare Outcomes) model for cancer screening training. ECHO provides structure for tele-mentoring providers through virtual sessions, including didactic and case-based learning formats. (11) Since its launch, Project ECHO has expanded to cover almost 50 
specialty areas and is currently conducted at 366 institutions in 39 countries (https://echo.unm.edu/locations). A recent review highlighted studies reflecting the widespread implementation of the model internationally. (12) The authors found, however, that there are very few studies that examine the impact of the ECHO model on provider behavior or health outcomes. The goal of this study is to examine the change in knowledge and skills among primary care physicians attending NICPR-ECHO trainings and the impact of the training program on implementation of cancer screening services.

\section{Methods}

\section{NICPR-ECHO model for cancer screening}

A detailed version of the NICPR-ECHO methods and the overall model related to training other health care providers (including dentists and gynecologists) has been published elsewhere.(13) This study focuses on primary care providers, and a short description of the ECHO curriculum is provided here to familiarize the reader with the training components. The training takes place in two phases - the first phase 14-week course with weekly online sessions; the second phase is a hands-on training that takes place at NICPR, approximately 1-3 months after the online phase. Table 1 outlines the content of the 14 online sessions along with the learning objectives per session.

\section{(Please insert Table 1 here)}

The NICPR team and other Indian experts developed the curriculum with a focus on the roles and responsibilities of primary care physicians in the population-based cancer screening program implementation.

The training modules include epidemiological data from GLOBOCAN (2) and involve both global and in-country experts during the didactic portion of the online sessions. Each session begins with a presentation by an expert for approximately 20-25 minutes, followed by case presentations by participants, and finally a discussion around questions that the participants may have. An aggregate of $80 \%$ score in the post-online phase assessment, participation in at least 10 sessions out of the 14, and presenting a case study are the minimum requirements for receiving a certificate and an invitation to a hands-on workshop.

The three-day hands-on workshops, conducted by NICPR in collaboration with national experts, focus on teaching trainees practical skills related to conducting the screening tests.. On the first day, trainees learn to prepare $5 \%$ acetic acid, insert a vaginal speculum, perform visual inspection with acetic acid (VIA), and document the test results. On the second day, trainees learn about the clinical breast examination (CBE) and how to perform it and record the results. The third day, they learn how to perform and document the Oral Visual examination (OVE) as well as communication skills and how to break bad news.

Although the training program does not provide continuing medical education credits, participants consider an ICMR certification of their attendance at the course very valuable in terms of their professional development. An aggregate of $80 \%$ score in the post-online phase assessment, participation in at least 10 sessions out of 14, and presenting a case study are the minimum requirement for receiving a certificate and an invitation to the handson workshop. A score of $80 \%$ on skills assessment of all three cancers makes the trainees eligible to receive a completion certificate for the hands-on phase. 


\section{Assessing training impact}

Figure 2 provides an overview of the NICPR-ECHO training strategy for primary care providers and the time points at which trainees undergo assessments. Prior to the start and again upon completion of the online phase, trainees answer a 23-item questionnaire, developed by NICPR-ECHO project team and administered online via SurveyMonkey. Table 2 provides an overview of the constructs measured and sample questions, and the full survey is available upon request (please contact the corresponding author). During the weekly sessions, trainees answer a short 5-item survey to assess their knowledge on the content of the session, both before and after each session, also via SurveyMonkey. At the end of the hands-on workshops, experts evaluate trainees for their skills in performing the screening tests for cervical, breast, oral cancers, and tobacco cessation counseling.

(Please insert Table 2 here)

\section{Data analysis}

\section{The data presented in this paper were analyzed using SPSS version 21 and primarily provide descriptive and bivariate analysis of the pre-post assessments of the online phase. To the extent possible, we provide descriptive data on the weekly sessions and the in- person workshops.}

\section{Results}

\section{Trainee characteristics}

Since the introduction of the NICPR-ECHO in 2016, 641 primary care physicians have participated in the trainings, across nine cohorts of trainees. This study presents data from 116 trainees (of the total 641 trainees) who completed both the pre- and post-assessments, representing 6 cohorts of trainees (trained from May 2019 to February 2020). Table 3 provides the demographics of the trainees, where available. Overall, $69 \%$ were male. Trainees were between age 26 to 63 years (average age $=39.2$ years). Almost two-thirds of trainees thirds had completed medical training (MD equivalent) $(69.7 \%$ ) and $85 \%$ worked in government healthcare facilities. Maharashtra in the western part of India, was the Indian State/Union Territory with the most trainees $(n=x$, $20.4 \%)$. Of the 116 trainees, 25 (21.6\%) participated in the in-person workshops. Workshop participants were between $25-55$ years of age, and approximately half $(n=13)$ were female. Similar to the online phase, the majority were medical graduates $(61.9 \%)$ and worked in government healthcare facilities $(n=20,80 \%)$. Among the Indian States/Union Territories, Tripura, in Eastern India, was the most common state of origin $(n=7,28 \%)$.

(Please insert Table 3 here) 
Additional file 1 shows the total number of respondents from six trained cohorts that answered the weekly quizzes. Since complete data was not available from all cohorts, we focused our analysis on cohort 9 that enrolled 114 trainees. To receive an invitation to the hands-on workshop, participants needed to score at least $80 \%$ on the weekly quizzes. On average, $35 \%$ of trainees scored above $80 \%$ on the post-weekly quizzes.

\section{Impact of the training on the knowledge of screening among participants}

Table 4 shows the overall change in knowledge scores between pre and post assessments for the online phase. Trainees reported statistically significant improvements when queried specifically on knowledge and skills using visual vignettes about oral and cervical cancer screening. Although there were changes in the positive direction for knowledge regarding breast cancer screening, these changes were not statistically significant. One question assessed confidence in cervical cancer screening, where there was a significant improvement in the confidence of trainees after the online phase (Mean=2.24, $S D=0.71)$ than before the training (Mean=1.22; $S D=0.10) ; t$ $(d f, 82)=9.57, p=0.000)$.

(Please insert Table 4 here)

Implementation of cancer screening services

When asked about their motivation to participate in the course, most trainees $(52.6 \%)$ reported wanting to gain the ability to implement screening in their practice settings. Only $30.2 \%$ noted that they wanted to gain more knowledge and the remaining approximately $16 \%$ wanted to become more confident in cancer screening. We also assessed their perception about implementation of cancer screening services in their practice settings. When queried about whether medical officers were currently providing cancer-screening services, the total percent of trainees reporting not screening at pre-test fell from $27.6 \%$ to $21.6 \%$ at post-assessment. For those who did not provide cancer screening on the post-test, $25.9 \%$ trainees reported the lack of consumables and equipment and $17.2 \%$ reported the lack of trained health care providers as reasons for not providing cancer screening services at their practice settings.

In an open text format, we also asked trainees to report other reasons why they did not provide cancer screening services at their settings. The most common reasons reported were ongoing prioritization of diabetes and hypertension screenings and providing cancer screening services in the form of camp-based community campaigns instead of during routine clinic visits. At the post-test, in an open text format, we also asked trainees to report on the total number of individuals they had screened. A crude analysis of these open-text data suggests approximate numbers of 12,766 individuals screened for breast cancer, 167,882 for oral cancer, and 4,025 for cervical cancer. To address the cancer control continuum, we asked the trainees whether they referred screen positive individuals to a tertiary center for diagnostic follow-up. Approximately $97 \%$ reported referring patients. Among those who did not refer, the most common reason noted was the referral center being too far for patients to reach. 


\section{Assessment of the hands-on workshop}

Additional file 2 contains the skills assessments tool that covers an assessment of all three cancers, breast, cervical, and oral. Experts and the NICPR team, at the end of the 3-day hands-on workshop, conduct the skills assessment using a pre-defined checklist. All 25 trainees (from the 116) scored at least an $80 \%$ score on their assessments and received a workshop completion certificate.

\section{Discussion}

The data collected from this study suggest that the NICPR-ECHO training program for primary care physicians improved the knowledge, skills, and confidence in delivering cancer-screening services according to the MoFHW guidelines, in the short term. These findings are similar to improvements reported among dentists and gynecologists undergoing similar training. $(14,15)$ The study, however, shows no statistical difference in primary care physicians' implementation of cancer screening services in their healthcare organizations, at the end of the 3-month training compared to when they enrolled in the program. This highlights a critical gap in promoting cancer prevention objectives in India and indicates the need for further research to explore the barriers to implementation of cancer screening services in the national and the regional and/or local context. The ECHO model is well suited to the Indian context in that it offers a virtual platform, is cost effective, and provides the ability to train physicians over a wide geographical spread in a limited time period. Globally, ECHO programs have shown evidence in improving provider knowledge and skills for treatment for several medical conditions such as Hepatitis C (12), Multiple Sclerosis (13), and behavioral disorders (14). Despite the growing number of studies evaluating $\mathrm{ECHO}$, a recent systematic review by McBain and colleagues concluded that the evidence of effectiveness was modest compared to the widespread implementation of the model internationally. (12) Specifically, the authors of the review raised an important question of whether the ECHO model could go beyond improving knowledge to actually influence provider behavior and build capacity for implementation of clinical services, a distinction which is supported by findings from this study.

Three specific considerations influence the implementation of an organized, population-based, cancer screening program in India. First, the proposed guidelines are to implement the cancer prevention program in an existing public healthcare delivery system that has historically been designed to provide maternal and child healthcare. However, maternal and child healthcare delivery have different objectives and functions than cancer screening programs (i.e. services are typically sought out by the patient, are limited to discrete time periods in a patient's life, do not typically need referral systems, and require different skill sets). A move to incorporate cancer prevention and control into this system will require implementation support (i.e. training, resources, staff, etc.) for primary care clinicians and their teams, including community health care workers since this is a new activity included in the public health system. Second, cancer screening presents a challenge for implementation because its success requires completion of several steps beyond the initial screening test (i.e. diagnostic testing, appropriate referrals, and treatment). Therefore, understanding the delivery of the screening process and available evidence regarding screening in the context of the healthcare, cultural, and geographical setting can be crucial for successful implementation and utilization of services. Third, the implementation of cancer screening programs can only be successful if providers are knowledgeable, skilled, and confident in delivery services and if healthcare systems are well resourced and ready for implementation with appropriate policy and stakeholder 
support. Aligning both the provider's and the healthcare organization's capacity requires a systematic implementation approach that is currently missing from the proposed organizational framework.

As noted above, a range of barriers may impede the behavioral change in health professionals, including the healthcare settings in which they practice. Change in clinical practice in low-, and middle-income countries requires the use of strategies that target either the health care organizations or healthcare workers. Very limited evidence currently exists on strategies that target healthcare organizations. (16) For strategies targeting health care workers, previous research suggests that compared to no intervention, internet based learning and educational workshops improve health workers knowledge, but it is unclear on whether such strategies improve health professionals' skills, behaviors, and whether this leads to improved patient outcomes. (16-18) However, interactive education (such as that delivered in NICPR-ECHO) or when combining interventions such as audit and feedback with educational meetings, are more effective than educational meetings alone Similarly, the use of interactive techniques (audit and feedback, academic detailing, outreach) are most effective for changing physicians care and patient outcomes.(19) Changes in healthcare professional behaviors and settings may happen if implementation strategies address specific barriers and tailored interventions have shown more effectiveness than no interventions or dissemination of guidelines alone.(20) Careful investigation of the context and the determinants is a perquisite for the implementation strategies to be effective in changing clinician behaviors and lead to practice change. Based on the current study findings and the given challenge to study the complexity, we must commit to the process of choosing strategies, developing and refining strategies, and studying the effectiveness of strategies that lead to implementation outcomes.(21) Successful implementation of cancer screening services through the public health system in India also requires the use of specific implementation theories, methods, and measures that can provide a systematic approach.

Parallel to other studies published on the NICPR-ECHO program, this study contributes findings showing improvements in knowledge noted in primary care physicians. $(14,15,22)$ We acknowledge the limitations of this study, which revolve around the pre-post, one group design for the evaluation of the ECHO program. Participants may have learned about cancer screening from other sources than the ECHO training, which could have influenced improvements noted on the post-test. Additionally, the testing itself may have affected the participant's responses at the post-test.

\section{Conclusion}

The NICPR-ECHO training model is an important first step towards the implementation of cancer screening services in India, per the national guidelines. Study finding support the effectiveness of the training program in reaching primary care physicians across the country and improving their knowledge and skills related to screening for breast, oral, and cervical cancer, which are significant public health problems in India and other low- and middle-income countries. After the training, very few primary care physicians mentioned implementing cancer screening services highlighting barriers that require further study and development of complementary implementation strategies. Study findings could inform the development and refinement of training for cancerscreening programs in low- and middle-income countries.

\section{Abbreviations}

ICMR-NICPR: Indian Council of Medical Research - National Institute of Cancer Prevention and Research 
ECHO: Extension for Community Healthcare Outcomes

MoHFW: Ministry of Health and Family Welfare

VIA: visual inspection with acetic acid

CBE: clinical breast examination

OVE: Oral Visual examination

MRI: Magnetic Resonance Imaging

FNAC: Fine Needle Aspiration Cytology

\section{Declarations}

\section{Ethics approval}

The Institutional Ethics Committee at the National Institute of Cancer Prevention and Research approved this study.

\section{Consent for publication}

Not applicable

\section{Availability of data and materials}

All data analyzed during this study are included in this article and are available from the corresponding author on reasonable request.

\section{Competing interests}

The authors declare they have no competing interests.

\section{Funding}

Tata Trusts funded our program from July 2018 to March 2020

\section{Authors contributions}

PA and RH conceptualized the article, interpreted the data, and drafted the article. KD, SN and SHC collected and analyzed the data, contributed to data interpretation along with providing substantial revisions to the article. All authors have approved the submitted manuscript. All authors have agreed both to be personally accountable for 
the author's own contributions and to ensure that questions related to the accuracy or integrity of any part of the work, even ones in which the author was not personally involved, are appropriately investigated, resolved, and the resolution documented in the literature.

\section{Acknowledgements}

We acknowledge the input of the participants included in this study and the statistical help provided by Mr. Vipin Kumar.

\section{References}

1. Dandona L, Dandona R, Kumar GA, Shukla D, Paul VK, Balakrishnan K, et al. Nations within a nation: variations in epidemiological transition across the states of India, 1990-2016 in the Global Burden of Disease Study. The Lancet. 2017;390(10111):2437-60.

2. Bray F, Ferlay J, Soerjomataram I, Siegel RL, Torre LA, Jemal A. Global cancer statistics 2018: GLOBOCAN estimates of incidence and mortality worldwide for 36 cancers in 185 countries. CA Cancer J Clin. 2018;68(6):394-424.

3. Welfare MoHaF. Operational Framework: Management of Common Cancers India2016 [Available from: http://cancerindia.org.in/cp/images/PDF/Operational_Framework_Management_of_Common_Cancers.pdf.

4. Hazarika I. Role of private sector in providing tuberculosis care: evidence from a population-based survey in India. Journal of global infectious diseases. 2011;3(1):19.

5. Mohanan M, Hay K, Mor N. Quality Of Health Care In India: Challenges, Priorities, And The Road Ahead. Health Affairs. 2016;35(10):1753-8.

6. (CBHI) CBoHI. National Health Profile 2015. 2015.

7. Khanna D, Khargekar N, Budukh A. Knowledge, attitude, and practice about cervical cancer and its screening among community healthcare workers of Varanasi district, Uttar Pradesh, India. J Family Med Prim Care. 2019;8(5):1715-9.

8. Singh E, Seth S, Rani V, Srivastava DK. Awareness of cervical cancer screening among nursing staff in a tertiary institution of rural India. J Gynecol Oncol. 2012;23(3):141-6.

9. Anandani C, Metgud R, Ramesh G, Singh K. Awareness of General Dental Practitioners about Oral Screening and Biopsy Procedures in Udaipur, India. Oral Health Prev Dent. 2015;13(6):523-30.

10. Singh GN, Agarwal A, Jain V, Kumar P. Understanding and Practices of Gynaecologists Related to Breast Cancer Screening, Detection, Treatment and Common Breast Diseases: A Study from India. World Journal of Surgery. 2019;43(1):183-91.

11. Arora S, Thornton K, Murata G, Deming P, Kalishman S, Dion D, et al. Outcomes of treatment for hepatitis C virus infection by primary care providers. New England Journal of Medicine. 2011;364(23):2199-207.

12. McBain RK, Sousa JL, Rose AJ, Baxi SM, Faherty LJ, Taplin C, et al. Impact of Project ECHO Models of Medical Tele-Education: a Systematic Review. Journal of general internal medicine. 2019;34(12):2842-57.

13. Babu R, Dhanasekaran K, Mehrotra R, Hariprasad R. Leveraging Technology for Nation-Wide Training of Healthcare Professionals in Cancer Screening in India: a Methods Article. Journal of Cancer Education. 
2020:1-7.

14. Nethan ST, Hariprasad R, Babu R, Kumar V, Sharma S, Mehrotra R. Project ECHO: a Potential Best-Practice Tool for Training Healthcare Providers in Oral Cancer Screening and Tobacco Cessation. J Cancer Educ. 2019.

15. Dhanasekaran K, Babu R, Kumar V, Mehrotra R, Hariprasad R. Capacity Building of Gynecologists in Cancer Screening Through Hybrid Training Approach. Journal of Cancer Education. 2019:1-7.

16. Pantoja T, Opiyo N, Lewin S, Paulsen E, Ciapponi A, Wiysonge CS, et al. Implementation strategies for health systems in low-income countries: an overview of systematic reviews. Cochrane Database of Systematic Reviews. 2017(9).

17. Cook DA, Levinson AJ, Garside S, Dupras DM, Erwin PJ, Montori VM. Internet-based learning in the health professions: a meta-analysis. Jama. 2008;300(10):1181-96.

18. Vaona A, Banzi R, Kwag KH, Rigon G, Cereda D, Pecoraro V, et al. E-learning for health professionals. Cochrane Database of Systematic Reviews. 2018(1).

19. Bloom BS. Effects of continuing medical education on improving physician clinical care and patient health: a review of systematic reviews. International journal of technology assessment in health care. 2005;21(3):380.

20. Baker R, Camosso-Stefinovic J, Gillies C, Shaw EJ, Cheater F, Flottorp S, et al. Tailored interventions to address determinants of practice. Cochrane Database of Systematic Reviews. 2015(4).

21. Powell BJ, Fernandez ME, Williams NJ, Aarons GA, Beidas RS, Lewis CC, et al. Enhancing the Impact of Implementation Strategies in Healthcare: A Research Agenda. Frontiers in Public Health. 2019;7(3).

22. Basu P, Meheus F, Chami Y, Hariprasad R, Zhao F, Sankaranarayanan R. Management algorithms for cervical cancer screening and precancer treatment for resource-limited settings. International journal of gynaecology and obstetrics: the official organ of the International Federation of Gynaecology and Obstetrics. 2017;138 Suppl 1:26-32.

\section{Tables}

Table 1. Content and format of the online phase for NICPR-ECHO 


\section{Online Session content Learning objectives \\ modules}

$\begin{array}{llll}\begin{array}{l}\text { Module 1 } \\ \text {-Cervical } \\ \text { cancer } \\ \text { screening }\end{array} & \begin{array}{l}\text { 1. Introduction to } \\ \text { NICPR-ECHO's } \\ \text { online Cancer } \\ \text { Screening course }\end{array} & \begin{array}{l}\text { Document the burden of cancer in India } \\ \text { prevention using screening, including risk and benefits }\end{array} \\ & \begin{array}{l}\text { Highlight the role of primary care physicians in the nations' cancer } \\ \text { screening program }\end{array}\end{array}$

2. Screening tests - Describe the burden and natural history of cervical cancer for cervical cancer cancer

Identify the causes, risk factors, signs and symptoms of cervical

Understand the role of screening tests and the risks and benefits

- Choose the appropriate frequency and the screening intervals for the screening tests

. Evaluate the impact of Human Papilloma Virus (HPV) vaccination on future screening in India

Discuss the operational framework for cervical cancer screening 3. Visual
Inspection with

. Review the anatomy \& physiology of transformation zone and Acetic Acid (VIA) squamous columnar junction

Discuss the principles of VIA, including the advantages and limitations of VIA

- Describe the step-by-step procedure of VIA, including the preparation of $5 \%$ acetic acid

- Interpret the results of a VIA test

4. Management for screen positive individuals
. Describe the types of ablative therapies (cryotherapy, thermal ablation)

5. Case scenarios in cervical cancer screening and management
- Assess eligibility of patients, including contraindications
- Evaluate resulting complications and follow-up
- Describe the types of excisional methods
- Assess eligibility criteria for treatment with excisional methods and evaluate follow up - Present and discuss cases pertaining to precancerous and cancerous cervical lesions
Module 2 - Breast Cancer Screening
6. Introduction to breast cancer 
- Review the role of follow up and referral in reducing the burden of cancer

. Discuss the operational framework for breast cancer screening in India

7. Screening tests . Identify the types of screening tests for breast cancer (triple for breast cancer assessment that includes ultrasound, mammography, and Magnetic Resonance Imaging (MRI))

- Describe the eligibility, advantages, and limitations, for ultrasound

- Describe the eligibility, advantages, and limitations, for mammography

- Describe the eligibility, advantages, and limitations, for MRI

- Diagnose and manage screen positives with Fine Needle Aspiration Cytology (FNAC)/ biopsy

8. Case scenarios in breast cancer

Present and discuss cases pertaining to precancerous and screening and management

\section{Module 3 - Oral cancer screening}

(1)
oral cancer
9. Introduction to

\section{cancerous breast lesions}

(n)

- Describe the burden of oral cancer in India

- Identify the causes, risk factors, signs, and symptoms, of oral cancer and the common sites of oral cancer

Review the role of tobacco in oral cancer

10. Screening tests for oral cancer
Review the common abnormalities of the oral cavity

Understand the screening tests for oral cancer, including the advantages and disadvantages

Understand the efficacy of oral examination

Describe the technique of oral examination India

Discuss the operational framework for oral cancer screening in
Review the details of Nicotine Replacement Therapy (gums,
patches, lozenges, and others) and Non-Nicotine therapy (Bupropion,

11. Tobacco Cessation: Counseling and follow up Varenicline, and others)

- Describe the target population, infrastructural requirements (including budget and capacity) for the overall program design

Highlight the importance and efficacy of mobile-Health in population-based tobacco cessation programs and training

12. Infection Control
Review the importance of following disinfection protocols for screening equipment including sterilizing screening equipment and disposal of used items

Describe the process of preparing disinfectants or $0.5 \%$ hypochlorite solution
13. Breaking bad news
Employ the empathetic communication methods to break the news of screen results in individuals that are positive 

results

14. Documenting cancer screening

- $\quad$ Discuss data collection for cancer screening according to the operational framework for India (including the person responsible for filing the proforma, frequency of reporting, rationale and purpose of data collection)

Table 2. Constructs measured and sample questions included in the pre and post assessment of the online phase 


\section{Concepts measured}

Knowledge about national cancer screening program (total of 4 questions)

\section{Sample questions}

Have you been oriented towards the recently released mandate of MoHFW for Management of Common Cancers?

As per operational framework guidelines of MoHFW released in August 2016 , screening for common cancers is done once in

What is the recommended age for screening for all common cancers in India, as per the Operational Framework Guidelines?

The most common cancer in Indian men is

Knowledge in screening for breast $(n=4)$, cervical $(n=4)$, and oral cancer $(n=5)$ (total 13 questions)
Confidence in performing screening tests (1 question)

Motivation to enroll in the course (1 question)

Implementation related questions (5 questions)
Breast related question: Diagnosis based on the clinical finding of the breast (image of breast showing orange peel appearance of breast, Peau d' orange) a) Dry skin; b) Peau de orange; c) Contact dermatitis; d) Mastitis

Cervical related questions: Interpretation of VIA test result (image of an acetowhite lesion in one quadrant of the cervix) a) Suspicious of malignancy; b) VIA positive; c) VIA negative; d) Squamous metaplasia

Oral related question: Diagnosis based on clinical finding in the oral mucosa (image of buccal mucosa showing a well-defined patch $1.5 \times 1$ $\mathrm{cm}$ with white \& erythematous areas, cracked mud surface appearance)

a) Leukoplakia; b) Lichen planus; c) Erythroplakia

d) Oral submucous fibrosis

How confident are you in performing cervical cancer screening?

What is the primary aim of you enrolling in this course? (may choose whichever is applicable)

Are you currently screening the people attending your health facility for cancer (oral, breast and cervix)?

If yes, do you counsel for tobacco cessation at your health facility?

How many individuals have you screened so far? (Please indicate for each of these cancers: oral, breast and cervical)

If you have started cancer screening, are you referring the screen positive individuals to higher facility for further management?

If screen positives are not referred, what is the reason?

Abbreviations used: MoHFW- Ministry of Health and Family Welfare

Table 3. Demographic characteristics of the trainees 


\begin{tabular}{|c|c|c|c|}
\hline \multicolumn{2}{|c|}{ Demographic characteristics } & $\begin{array}{l}\text { Online phase } \\
(\mathrm{N}=116) \\
\mathrm{n}(\%)\end{array}$ & $\begin{array}{l}\text { Hands-on } \\
\text { workshop }(\mathrm{N}=25) \\
\mathrm{n}(\%)\end{array}$ \\
\hline \multirow{4}{*}{ Gender } & & & \\
\hline & Male & $81(69.8)$ & $12(48.0)$ \\
\hline & Female & $35(30.1)$ & $13(52.0)$ \\
\hline & Total & $116(100.0)$ & $25(100.0)$ \\
\hline \multirow{5}{*}{$\begin{array}{l}\text { Age, in } \\
\text { years }\end{array}$} & $25-35$ & $19(38.0)$ & $2(40.0)$ \\
\hline & $36-45$ & $17(34.0)$ & $1(20.0)$ \\
\hline & $46-55$ & $13(26.0)$ & $2(40.0)$ \\
\hline & $>55$ & $1(2.0)$ & $0(00.0)$ \\
\hline & Total & $50(100)^{\star}$ & $5(100)^{\star}$ \\
\hline \multirow[t]{3}{*}{ Education } & Graduates (MD equivalent) & $72(70.5)$ & $13(61.9)$ \\
\hline & Post graduates & $30(29.4)$ & $8(38.0)$ \\
\hline & Total & $102(100.0)^{\star}$ & $21(100.0)^{\star}$ \\
\hline \multirow{3}{*}{$\begin{array}{l}\text { Practice } \\
\text { setting }\end{array}$} & Government & $99(85.3)$ & $20(80.0)$ \\
\hline & Private & $17(14.6)$ & $5(20.0)$ \\
\hline & Total & $116(100.0)$ & $25(100.0)$ \\
\hline \multirow[t]{11}{*}{ Region } & North region & $21(18.1)$ & $4(16.0)$ \\
\hline & $\begin{array}{l}\text { (includes Delhi, Haryana, Jammu and Kashmir, Punjab, } \\
\text { Rajasthan, Uttar Pradesh) }\end{array}$ & & \\
\hline & South region & $15(12.9)$ & $6(24.0)$ \\
\hline & $\begin{array}{l}\text { (includes Andhra Pradesh, Karnataka, Kerala, } \\
\text { Puducherry) }\end{array}$ & & \\
\hline & East region & $35(30.1)$ & $9(36.0)$ \\
\hline & (includes Assam, Bihar, Odisha, West Bengal, Tripura) & & \\
\hline & West region & $31(26.7)$ & $3(12.0)$ \\
\hline & $\begin{array}{l}\text { (includes Dadar and Nagar Haveli, Goa, Gujrat, } \\
\text { Maharashtra) }\end{array}$ & & \\
\hline & Central region & $14(12.0)$ & $3(12.0)$ \\
\hline & (includes Chhattisgarh, Madhya Pradesh) & & \\
\hline & Total & $116(100)$ & $25(100.0)$ \\
\hline
\end{tabular}

*Information not available for all participants, since they chose not to answer these questions in the survey 
Table 4. Change in knowledge, skills, and confidence in cancer screening among primary care physicians that trained in the online phase

\begin{tabular}{|llll|}
\hline Concepts measured (no. of items) & $\begin{array}{l}\text { Pre- } \\
\text { assessment } \\
\text { Mean (SD) }\end{array}$ & $\begin{array}{l}\text { Post-assessment Mean } \\
\text { (SD) }\end{array}$ & $\begin{array}{l}\text { p } \\
\text { value }\end{array}$ \\
\hline Overall knowledge (16 items) & $8.2(1.86)$ & $9.8(1.91)$ & $0.000^{\star}$ \\
\hline $\begin{array}{l}\text { Knowledge about national cancer screening program } \\
\mathbf{4} \text { items) }\end{array}$ & $2.8(0.96)$ & $3.4(0.72)$ & $0.000^{\star}$ \\
\hline Knowledge in screening for oral cancer (5 items) & $2.9(0.93)$ & $3.4(1.06)$ & $0.000^{\star}$ \\
\hline Knowledge in screening for breast cancer (4 items) & $1.8(0.88)$ & $1.7(0.88)$ & 0.353 \\
\hline Knowledge in screening for cervical cancer (4 items) & $1.7(0.84)$ & $2.1(0.88)$ & $0.000^{\star}$ \\
\hline
\end{tabular}

${ }^{*} p<0.001$, statistically significant results on a paired t-test

\section{Figures}






Figure 1

Public Health Infrastructure in India 


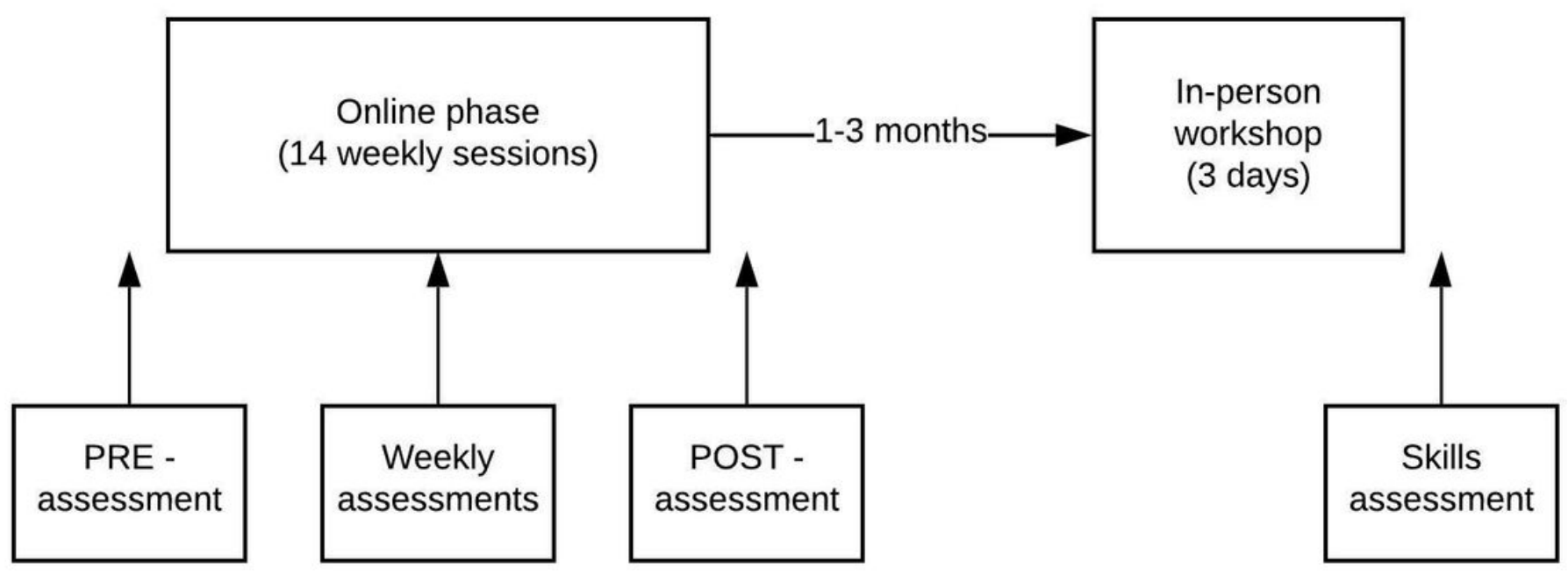

Figure 2

Overview of the training strategy and assessment

\section{Supplementary Files}

This is a list of supplementary files associated with this preprint. Click to download.

- Additionalfile1.docx

- Additionalfile2.docx 